\title{
Portable Infrared Pupillometer in Patients With Subarachnoid Hemorrhage: Prognostic Value and Circadian Rhythm of the Neurological Pupil Index (NPi)
}

\author{
Natzeder, Samuel ; Mack, David J ; Maissen, Gabriela ; Straessle, Christian ; Keller, Emanuela ; Muroi, Carl
}

\begin{abstract}
Background: Portable automated infrared pupillometry is becoming increasingly popular. To generate an objective reference base, the Neurological Pupil index (NPi) which combines different values of the pupillary light reflex is being introduced into clinical practice. In this explorative study, we examined different aspects of the NPi in relation to clinical severity and outcome in patients with aneurysmal subarachnoid hemorrhage (aSAH). Materials and Methods: Patients with serial assessment of the NPi (NeurOptics pupillometer NPi-200, Irvine, CA) starting no later than day 2 after aSAH onset were included in the study. Relative numbers of pathologic NPi's, absolute NPi values, and their variances were compared according to aSAH clinical severity grade, functional outcome, and case fatality. The correlation between NPi and intracranial pressure, and NPi periodicity, were also examined. Results: In total, 18 patients with $4456 \mathrm{NPi}$ values were eligible for inclusion in the analysis. The general trend of the NPi over time reflected the course of the neurological illness. Mean NPi tended to be lower in patients with clinically severe compared with nonsevere aSAH (3.75 \pm 0.40 vs. $4.56 \pm 0.06$; $\mathrm{P}=0.171)$, and in patients with unfavorable compared with favorable outcomes ( $3.64 \pm 0.48$ vs. $4.50 \pm 0.08 ; \mathrm{P}=0.198)$. The mean variance of the NPi was higher in patients with severe compared with nonsevere aSAH $(0.49 \pm 0.17 \mathrm{vs}$. $0.06 \pm 0.02 ; \mathrm{P}=0.025)$. Pathologic NPi values were recorded more frequently in patients with severe compared with nonsevere aSAH $(16.3 \% \pm 8.8 \%$ vs. $0.0 \% \pm 0.0 \% ; \mathrm{P}=0.002)$, and in those with unfavorable compared with favorable outcomes $(19.2 \% \pm 10.6 \%$ vs. $0.7 \% \pm 0.6 \% ; \mathrm{P}=0.017)$. NPi was inversely correlated with intracranial pressure (Spearman $\mathrm{r}=-0.551, \mathrm{P}<0.001)$. We observed a circadian pattern of NPi's which was seemingly disrupted in patients with fatal outcome. Conclusions: On the basis of this preliminary study, the assessment of NPi by pupillometry is feasible and might complement multimodal neuromonitoring in patients with aSAH.
\end{abstract}

DOI: https://doi.org/10.1097/ANA.0000000000000553

Posted at the Zurich Open Repository and Archive, University of Zurich

ZORA URL: https://doi.org/10.5167/uzh-168482

Journal Article

Published Version

Originally published at:

Natzeder, Samuel; Mack, David J; Maissen, Gabriela; Straessle, Christian; Keller, Emanuela; Muroi, Carl (2019). Portable Infrared Pupillometer in Patients With Subarachnoid Hemorrhage: Prognostic Value and Circadian Rhythm of the Neurological Pupil Index (NPi). Journal of Neurosurgical Anesthesiology, 31(4):428-433.

DOI: https://doi.org/10.1097/ANA.0000000000000553 


\title{
Portable Infrared Pupillometer in Patients With Subarachnoid Hemorrhage: Prognostic Value and Circadian Rhythm of the Neurological Pupil Index (NPi)
}

\author{
Samuel Natzeder, MMed ${ }^{1}$, David J. Mack, $\mathrm{PhD}^{1}$, Gabriela Maissen, MMed ${ }^{1}$, Christian Strässle $^{1}$, Emanuela \\ Keller, $\mathrm{MD}^{1}$, and Carl Muroi, $\mathrm{MD}^{1}$ \\ ${ }^{1}$ Neurocritical Care Unit, University Hospital Zurich, 8091 Zurich, Switzerland
}

Background: Portable automated infrared pupillometry is becoming increasingly popular. To generate an objective reference base, the Neurological Pupil index (NPi) which combines different values of the pupillary light reflex is being introduced into clinical practice. In this explorative study, we examined different aspects of the NPi in relation to clinical severity and outcome in patients with aneurysmal subarachnoid hemorrhage (aSAH).

Materials and Methods: Patients with serial assessment of the NPi (NeurOptics pupillometer NPi-200, Irvine, CA) starting no later than day 2 after aSAH onset were included in the study. Relative numbers of pathologic NPi's, absolute NPi values, and their variances were compared according to aSAH clinical severity grade, functional outcome, and case fatality. The correlation between NPi and intracranial pressure, and NPi periodicity, were also examined.

Results: In total, 18 patients with $4456 \mathrm{NPi}$ values were eligible for inclusion in the analysis. The general trend of the NPi over time reflected the course of the neurological illness. Mean NPi tended to be lower in patients with clinically severe compared with nonsevere aSAH ( $3.75 \pm 0.40$ vs. $4.56 \pm 0.06 ; P=0.171)$, and in patients with unfavorable compared with favorable outcomes ( $3.64 \pm 0.48$ vs. $4.50 \pm 0.08 ; P=0.198$ ). The mean variance of the NPi was higher in patients with severe compared with nonsevere aSAH $(0.49 \pm 0.17 \mathrm{vs}$. $0.06 \pm 0.02 ; \mathrm{P}=0.025)$. Pathologic NPi values were recorded more frequently in patients with severe compared with nonsevere aSAH $(16.3 \% \pm 8.8 \%$ vs. $0.0 \% \pm 0.0 \% ; \mathrm{P}=0.002)$, and in those with unfavorable compared with favorable outcomes $(19.2 \% \pm 10.6 \%$ vs. $0.7 \% \pm 0.6 \% ; P=0.017)$. NPi was inversely correlated with intracranial pressure (Spearman $r=-0.551, \mathrm{P}<0.001$ ). We observed a circadian pattern of NPi's which was seemingly disrupted in patients with fatal outcome.

Conclusions: On the basis of this preliminary study, the assessment of NPi by pupillometry is feasible and might complement multimodal neuromonitoring in patients with aSAH.

Key Words: Neurological Pupil index, pupillometer, subarachnoid hemorrhage, pupillary light reflex, intracranial pressure, circadian rhythm

Portable automated infrared pupillometry is becoming increasingly popular. Automated pupillometer data are claimed to be exact and have a better interrater reliability than conventional manual assessment, and hence provide superior clinical assessment as well as objective data. Infrared pupillometry is able to detect a pupillary light reflex (PLR) even when the pupil has been assessed as nonreactive by clinicians using standard methods. ${ }^{1-7}$

The Neurological Pupil index (NPi) has been developed as a composite value. It incorporates multiple variables, such as minimal and maximal pupil sizes, constriction velocity and latency, into a single-variable calculated using an algorithm developed by the manufacturer. ${ }^{2,6,8-12}$ It is claimed that the NPi is less vulnerable to confounding factors such as different ambient light conditions than standard methods of 
assessing pupil reactivity, and is reportedly independent of pupil size. ${ }^{13}$ The NPi might, therefore, have potential to predict critical events such as pathologic increases in intracranial pressure (ICP). ${ }^{9,14}$

However, the NPi cannot yet be regarded as a standard and the literature on its clinical applications is still sparse. Little is known about the relationship of NPi with neurological conditions, its predictive value, and the meaning of its dynamics. The aim of this explorative study was to examine the different aspects of the $\mathrm{NPi}$ in relation to clinical severity and outcome in patients with aneurysmal subarachnoid hemorrhage (aSAH).

\section{MATERIALS AND METHODS}

For this exploratory study, we retrospectively retrieved data from a prospectively collected database of aSAH patients treated at the Neurocritical Care Unit, University Hospital Zurich, between November 2016 and 2017. Patients' baseline characteristics and aSAH clinical severity grade assessed according to the World Federation of Neurosurgical Societies (WFNS) grading system were recorded. ${ }^{15}$ Clinical outcome at the time of hospital discharge was assessed according to the Glasgow outcome scale (GOS). ${ }^{15} \mathrm{~A}$ detailed description of the study methodology, including inclusion and exclusion criteria, is available in the supplementary material (Supplementary Methods and Results, Supplemental Digital Content 1, http://links.Iww.com/JNA/A76). NPi measurements were obtained by the NeurOptics pupillometer NPi-200 (NeurOptics, Irvine, CA). Pathologic NPi's were defined as $<3$, and a pathologic side difference as $\Delta N P i \geq 0.7$, as previously described. ${ }^{9}$

\section{Statistical Analysis}

Continuous variables are presented as mean \pm SE unless otherwise stated. Clinical severity was dichotomized into nonsevere (WFNS, 1 to 3 ) and severe (WFNS, 4 to 5) aSAH, and clinical outcome into favorable (GOS, 4 to 5 ) and unfavorable (GOS, 1 to 3 ). The relative number of pathologic NPi's, mean NPi values, and NPi variance were compared between patients with nonsevere and severe aSAH and favorable and unfavorable outcomes. Because of the small sample size, it was not feasible to reliably test for normality. Therefore, the nonparametric Mann-Whitney $U$ test was applied. We chose an alpha level of 0.05 to test for significance. As the number of consecutive days on which NPi was measured varied among patients, 4 time blocks were created for the analysis: days 0 to 3,4 to 6,7 to 9 , and 10 to 12 after aSAH. In patients where continuous ICP monitoring data were available, the relationship between ICP and NPi was assessed (Supplemental Material 1, Supplemental Digital Content 1, http://links.Iww.com/JNA/A76). We analyzed the periodicity of NPi values in a subgroup of patients in whom the NPi was recorded until at least day 10 (Supplementary Methods and Results, Supplemental Digital Content 1, http://links.Iww.com/JNA/A76).

\section{RESULTS}

A total of 18 patients were eligible for analysis, including 4456 NPi recordings. The average number of NPi values per patient was $248 \pm 37$. Patients' baseline characteristics and outcomes are shown in the supplementary material (Supplementary Table 1, Supplemental Digital Content 2, http://links.Iww.com/JNA/A77).

The absolute number of NPi measurements was significantly higher in patients with clinically severe compared with nonsevere aSAH ( $328 \pm 38$ vs. $121 \pm 40$; $P=0.003$ ). The cumulative number of days on which NPi measurements were made was also higher, although this difference was not statistically significant $(9.3$ \pm 0.8 vs. $6.9 \pm 1.4 \mathrm{~d} ; \mathrm{P}=0.169$ ). The number of NPi measurements was not significantly higher in patients with unfavorable outcome or on those who died in hospital (Supplementary Methods and Results, Supplemental Digital Content 1, http://links.Iww.com/JNA/A76). 
Mean NPi tended to be lower in patients with clinically severe compared with nonsevere aSAH $(3.75 \pm 0.40$ vs. $4.56 \pm 0.06 ; \mathrm{P}=0.171)$, and in patients with unfavorable compared with favorable outcomes $(3.64 \pm 0.48$ vs. $4.50 \pm 0.08 ; \mathrm{P}=0.198)$. This difference was more pronounced in patients who died in hospital compared with those who survived $(3.03 \pm 1.01$ vs. $4.37 \pm 0.12 ; P=0.075)$. Essentially, the mean NPi in patients with clinically severe aSAH was close to the pathologic threshold. A subgroup analysis of patients with clinically severe aSAH identified a significant lower mean NPi in those with a fatal outcome ( $2.5 \pm 1.2$ vs. $4.2 \pm 0.2$; $\mathrm{P}$ $=0.041)$. Patients with delayed cerebral ischemia $(n=7)$ also had a significantly lower mean NPi compared with those who did not $(3.9 \pm 0.2$ vs. $4.2 \pm 0.4 ; P=0.011)$.

The mean variance of the NPi was significantly higher in patients with clinically severe compared with nonsevere aSAH $(0.49 \pm 0.17$ vs. $0.06 \pm 0.02 ; \mathrm{P}=0.025)$. There was also a (nonsignificant) tendency toward higher variance in patients with unfavorable compared with unfavorable outcome $(0.53 \pm 0.21 \mathrm{vs}$. $0.11 \pm$ $0.04 ; \mathrm{P}=0.075)$. The variance difference between patients who did and did not die in hospital was not significant ( $0.64 \pm 0.43$ vs. $0.23 \pm 0.08 ; P=0.268$ ). Qualitatively, the trend of NPi values over time reflected the course of the neurological illness, and 2 illustrative cases are shown in Figure 1. Absolute pupil size and the relative number of anisocoria were not significantly different in any of the comparisons (Supplementary Methods and Results, Supplemental Digital Content 1, http://links.Iww.com/JNA/A76).

Pathologic NPi values were recorded more frequently in patients with severe compared with nonsevere aSAH ( $16.3 \% \pm 8.8 \%$ vs. $0.0 \% \pm 0.0 \% ; \mathrm{P}=0.002)$, and in those with unfavorable compared with favorable outcomes $(19.2 \% \pm 10.6 \%$ vs. $0.7 \% \pm 0.6 \% ; P=0.017)$. The relative number of pathologic NPi values was higher in patients who died in hospital compared with those who survived, but this difference was not statistically significant $(33.2 \% \pm 22.6 \%$ vs. $3.3 \pm 2.0 \%$; $P=0.203$ ). There was a higher relative number of occurrences of a pathologic between-side difference in patients with severe aSAH, unfavorable outcome, and in-hospital death compared with those with nonsevere aSAH, favorable outcome, and in-hospital survival, respectively (data not shown).

Analysis of the predefined time blocks showed that the number of pathologic NPi values on days 0 to 3 was significantly higher in patients with severe aSAH, unfavorable outcome, and in-hospital mortality compared with those with nonsevere aSAH, favorable outcome and in-hospital survival, respectively $(10.3 \% \pm 8.2 \%$ vs. $0.0 \% \pm 0.0 \%, P=0.038 ; 12.6 \% \pm 10.1 \%$ vs. $0.0 \% \pm 0.0 \%, P=0.009 ; 24.3 \% \pm 22.7 \%$ vs. $1.2 \% \pm 0.9 \%, P=0.044$, respectively). Although this tendency was also be observed in the other time blocks, these differences were not statistically significant except between patients with favorable and unfavorable outcome on days 7 to 9 $(35.3 \% \pm 16.8 \%$ vs. $0.7 \% \pm 0.7 \% ; P=0.042)$. As shown in Figures $2 A-C$, the mean NPi values tended to be lower in patients with clinically severe hemorrhage, unfavorable outcome, and in-hospital mortality, with the differences seemingly most pronounced on days 7 to 9 . However, a statistically significant difference between patients who died in hospital and those who survived was observed only on days 4 to 6 and days 7 to 9. The variance in NPi values tended to be higher in patients with clinically severe aSAH, unfavorable outcome and in-hospital mortality and, although the differences were again more pronounced on days 7 to 9, there was no statistical significance at any timepoint (Figs. 2D-F). There were also no noteworthy differences in the betweenside differences in number of pathologic NPi between any of the time blocks (data not shown).

There was an inverse correlation between NPi and ICP (Fig. 3A). The confusion matrix revealed that the sensitivity of $\mathrm{NPi}<3$ to detect a pathologic ICP $(>15 \mathrm{mmHg})$ was $35.5 \%$. The NPi performed better in ruling out a pathologic ICP with a specificity of $95.7 \%$. The subgroup analysis of patients with measurements for $>10$ consecutive days showed a circadian cyclic rhythm of the NPi (Figs. 1B, C, 3B, C). The relative power sum in the circadian frequency was lower in patients who died in hospital than in those who survived (Fig. 3D). Although this finding was not statistically significant, it might indicate a disrupted circadian rhythm in patients with fatal outcome. 


\section{DISCUSSION}

The results of this exploratory analysis suggest that the NPi reflects the clinical severity of aSAH and that pathologic NPi values are associated with a poor clinical outcome. Besides the absolute NPi value, its variance and general trends are also likely associated with both clinical severity and outcome. Last but not least, we found an inverse correlation between NPi and ICP, as previously described. ${ }^{9}$

Therefore, pupillary status as quantified by the NPi might be a useful parameter to estimate the severity of aSAH as well as an additional monitoring parameter for the detection of secondary deterioration and prognostication.

Several grading systems-based either on clinical or radiologic criteria-have been proposed for outcome prediction or decision support after $\mathrm{aSAH},{ }^{15}$ and more recently proposed systems have attempted to combine the two. Clinical grading systems can be troublesome because a considerable proportion of patients with aSAH are intubated or sedated, thereby restricting clinical assessment. In this context, pupillary status might be an ideal parameter by which severity of aSAH can be assessed. However, pupil status is not currently incorporated into any aSAH grading system. Two previous studies have focused on the predictive value of initial pupillary status in patients with severe aSAH. ${ }^{16,17}$ In these studies, the PLR was assessed conventionally and categorized as bilaterally reactive, unilaterally reactive or bilaterally nonreactive, and absent pupillary reactivity was associated with poor clinical outcomes in both. Our results showed that the relative number of pathologic NPi's measured in the early phase (day 0 to 3 after ictus) was associated with poor outcome, a finding that supports the assumption that disturbance of the pupillary function in the early phase after aSAH might be a useful predictive tool. Therefore, we propose that consideration be given to incorporating NPi into outcome prediction algorithms.

Besides the initial impact of the hemorrhage, patients with aSAH are at risk of developing secondary brain damage. Classically, cerebral vasospasm was considered to be the main cause but recent evidence points to a more complex and multifactorial etiology. Detecting imminent secondary brain injury remains a challenge in contemporary neurocritical care. Generally patients are at risk between day 4 and 12 post-aSAH, ${ }^{15}$ with a peak around day 7 and 9. However, patients, particular those with severe aSAH, are often comatose or sedated, thereby limiting neurological assessment. Multimodal neuromonitoring is recommended in such patients. As the general trend of the NPi followed the clinical course in our study, and NPi was inversely correlated with ICP, its inclusion as an additional multimodal neuromonitoring variable might be of value. However, the feasibility of NPi measurements in conscious patients is restricted, as it is not reasonable to expose them to the flashlights on a frequent basis. Even more problematic is its assessment in agitated or confused patients, as the subject must keep still during the pupillometry examination. ${ }^{5}$ In retrospect, our data clearly mirror this problem-good grade aSAH patients, who were less likely comatose, had lower absolute numbers of NPi measurements and days on which such measurements were made.

The influence of the endogenous circadian clock on the PLR has recently been described in healthy humans. ${ }^{18,19} \mathrm{~A}$ new and interesting observation in our study is the identification of a circadian rhythm in the NPi after aSAH, with a positive peak at midnight and negative peak at noon. Spectral analysis indicated that patients with fatal outcome had a less preserved circadian pattern, leading us to speculate that disruption of the circadian rhythm might be a measure of the extent of brain dysfunction and/or damage. As confounders such as ambient light conditions were not considered in our study, these findings must be interpreted with caution. However, the circadian pattern of NPi is an interesting observation which warrants further investigation. Little is currently known about circadian system status and its implication in the acute phase after an aSAH.

As this study is purely explorative, it has several methodological flaws and low statistical power. The number of patients was small and there was substantial heterogeneity concerning the assessed time periods. Importantly, continuous "long-term" (up to $10 \mathrm{~d}$ ) NPi measurements were only available in clinically severe cases. Another shortcoming is the fact that concurrent medication such as opioids and 
anesthetics/sedatives were not considered in the analysis. However, in contrast to what is widely believed, commonly used drugs in usual therapeutic doses do not severely depress PLR. ${ }^{13,20}$ In conclusion, assessment of NPi using the portable NeurOptics pupillometer NPi-200 is feasible and adds useful information in the setting of multimodal neuromonitoring after aSAH, especially in patients who are unconscious and/or under general anesthesia. Besides absolute NPi values, its variance and circadian pattern might provide additional information for prognostication. 


\section{REFERENCES}

1. Kerr RG, Bacon AM, Baker LL, et al. Underestimation of pupil size by critical care and neurosurgical nurses. Am J Crit Care. 2016; 25:213-219.

2. Couret D, Boumaza D, Grisotto C, et al. Reliability of standard pupillometry practice in neurocritical care: an observational, doubleblinded study. Crit Care. 2016;20:99.

3. Olson DM, Stutzman S, Saju C, et al. Interrater reliability of pupillary assessments. Neurocrit Care. 2016;24:251-257.

4. Zhao W, Stutzman S, DaiWai O, et al. Inter-device reliability of the NPi-100 pupillometer. J Clin Neurosci. 2016;33:79-82.

5. Meeker M, Du R, Bacchetti P, et al. Pupil examination: validity and clinical utility of an automated pupillometer. J Neurosci Nurs. 2005; 37:34-40.

6. Larson MD, Muhiudeen I. Pupillometric analysis of the "absent light reflex". Arch Neurol. 1995;52:369372.

7. Olson DM, Fishel M. The use of automated pupillometry in critical care. Crit Care Nurs Clin North Am. 2016;28:101-107.

8. Taylor WR, Chen JW, Meltzer H, et al. Quantitative pupillometry, a new technology: normative data and preliminary observations in patients with acute head injury. Technical note. J Neurosurg. 2003; 98:205-213.

9. Chen JW, Gombart ZJ, Rogers S, et al. Pupillary reactivity as an early indicator of increased intracranial pressure: the introduction of the Neurological Pupil index. Surg Neurol Int. 2011;2:82.

10. Chen JW, Vakil-Gilani K, Williamson KL, et al. Infrared pupillometry, the Neurological Pupil index and unilateral pupillary dilation after traumatic brain injury: implications for treatment paradigms. Springerplus. 2014;3:548.

11. Boev AN, Fountas KN, Karampelas I, et al. Quantitative pupillometry: normative data in healthy pediatric volunteers. J Neurosurg. 2005; 103:496-500.

12. McLaren JW, Hauri PJ, Lin SC, et al. Pupillometry in clinically sleepy patients. Sleep Med. 2002;3:347352.

13. Larson MD, Behrends M. Portable infrared pupillometry: a review. Anesth Analg. 2015;120:1242-1253.

14. McNett M, Moran C, Janki C, et al. Correlations between hourly pupillometer readings and intracranial pressure values. J Neurosci Nurs. 2017; 49:229-234.

15. Macdonald RL, Schweizer TA. Spontaneous subarachnoid haemorrhage. Lancet. 2017; 389:655-666.

16. Mack WJ, Hickman ZL, Ducruet AF, et al. Pupillary reactivity upon hospital admission predicts long-term outcome in poor grade aneurysmal subarachnoid hemorrhage patients. Neurocrit Care. 2008; 8:374-379.

17. Yoshimoto $\mathrm{Y}$, Wakai $\mathrm{S}$, Ochiai $\mathrm{C}$, et al. Significance of pupillary reactivity in poor-grade aneurysm patients as a prognostic factor and an indication for active treatment. Br J Neurosurg. 1997;11:25-31.

18. Bonmati-Carrion MA, Hild K, Isherwood C, et al. Relationship between human pupillary light reflex and circadian system status. PLoS One. 2016;11:e0162476.

19. Munch M, Leon L, Crippa SV, et al. Circadian and wake-dependent effects on the pupil light reflex in response to narrow-bandwidth light pulses. Invest Ophthalmol Vis Sci. 2012;53:4546-4555. 
20. Shirozu K, Setoguchi H, Tokuda K, et al. The effects of anesthetic agents on pupillary function during general anesthesia using the automated infrared quantitative pupillometer. J Clin Monit Comput. 2017;31:291-296. 
Figure 1. Continuous NPi measurements of two illustrative cases. A) Case 1, 38 years old female patient with aSAH, WFNS 4 . The general trend (superimposed line) shows a decline starting at day 3 , with a negative peak around day 7 and 8 . Clinically, the patient developed cerebral vasospasm at day 3 . The following days, the patients' condition declined further with additional development of cerebral edema. The condition slowly started to improve around day 8. Note the circadian pattern of the NPi. B) Case 2, 39 years old female patient with aSAH, WFNS 5 . The general trend shows a continuous decline beginning at day 4. Clinically, the patient developed as well cerebral vasospasm as brain edema at day 5 . The patients' condition declined further. Due to a concomitant sepsis, the cerebral perfusion pressure could not be maintained. At day 8, persistent vasospasm and beginning demarcations of ischemic infarcts were visible in a CT scan. Exitus letalis at day 10. Note, the circadian pattern is disrupted, in particular after day 3.

A

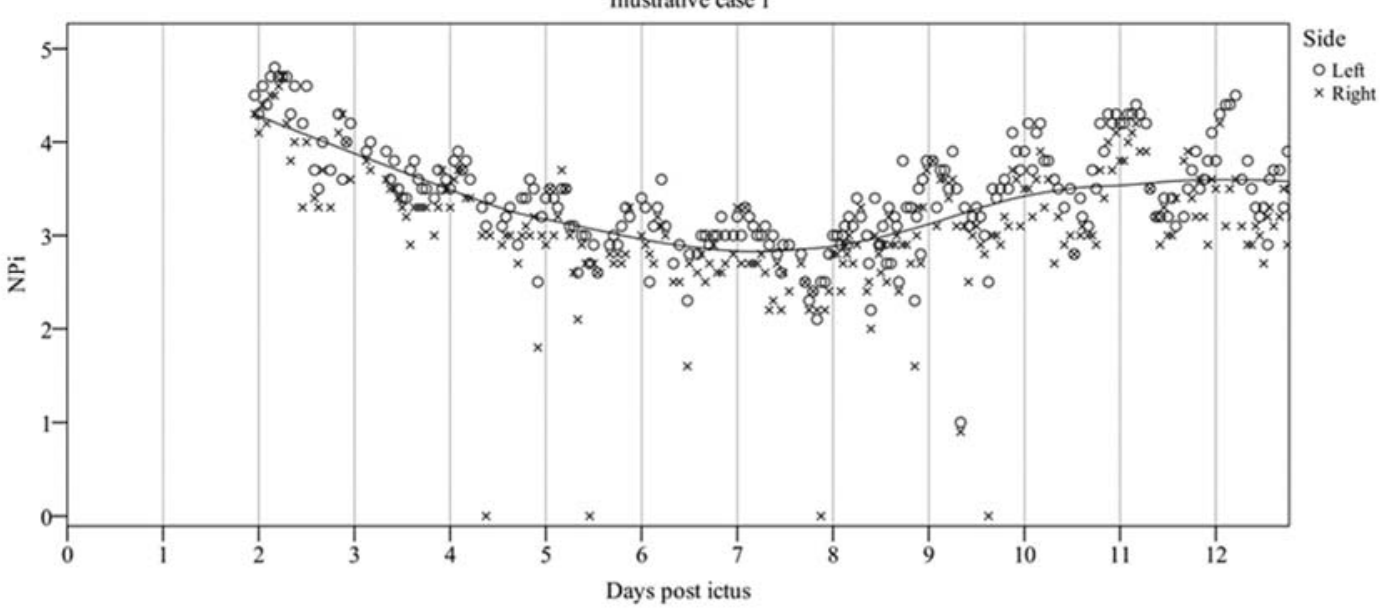

B

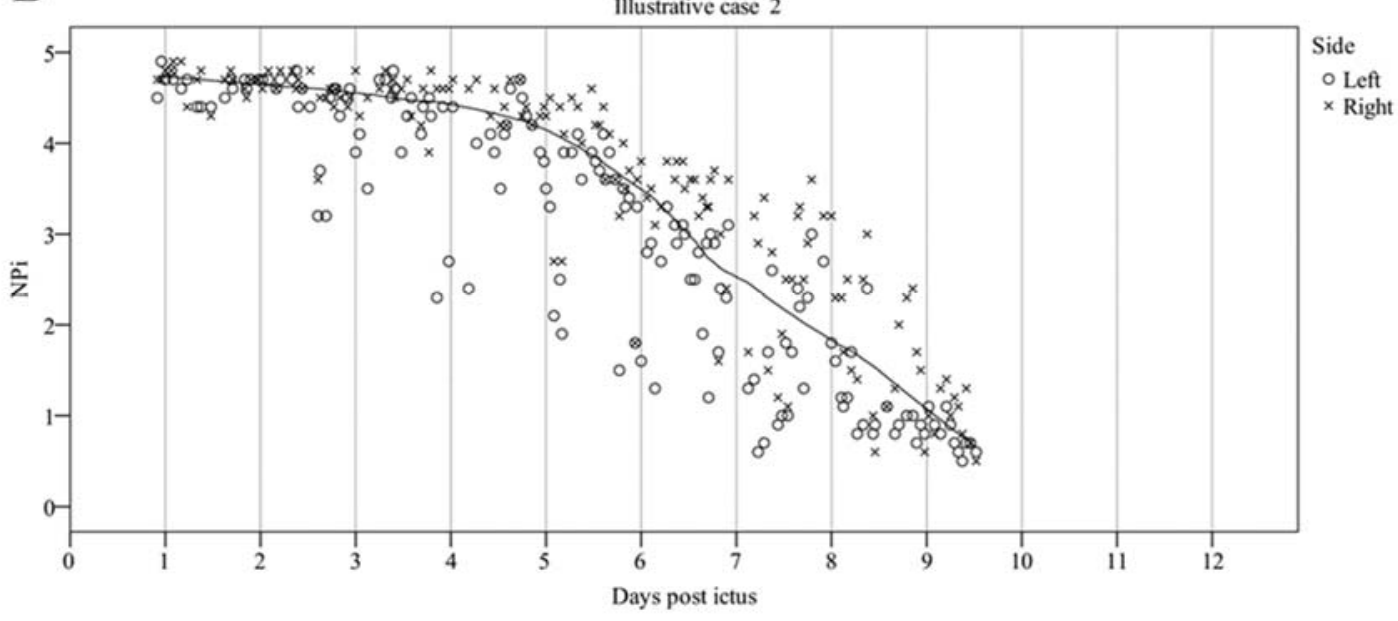


Figure 2. A) Mean NPi values within predefined time blocks stratified by clinical severity (WFNS), B) by outcome (GOS), and C) by in-hospital mortality. The dotted line represents the trend of patients with WFNS 1-3, GOS 4-5, and without in-hospital mortality, the continuous line the trend of patients with WFNS 4-5, GOS 1-3, and with in-hospital mortality respectively. Note the negative peak in the time block day 7-9. D) Mean variances in NPi values within predefined time blocks stratified by clinical severity (WFNS), E) by outcome (GOS), and F) by in-hospital mortality. The dotted line represents the trend of patients with WFNS 1-3, GOS 4-5, and without in-hospital mortality, the continuous line the trend of patients with WFNS 4-5, GOS 1-3, and with in-hospital mortality respectively. * statistically significant, $p=0.048$ at day $4-6$ and $p=0.018$ at day $7-9 ;+$ as only one patient was available in one group, statistical comparison was performed and the trend-line was discontinued.

A

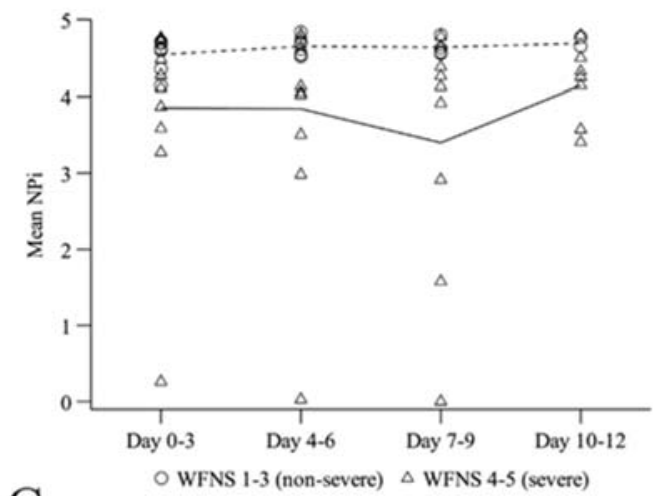

C

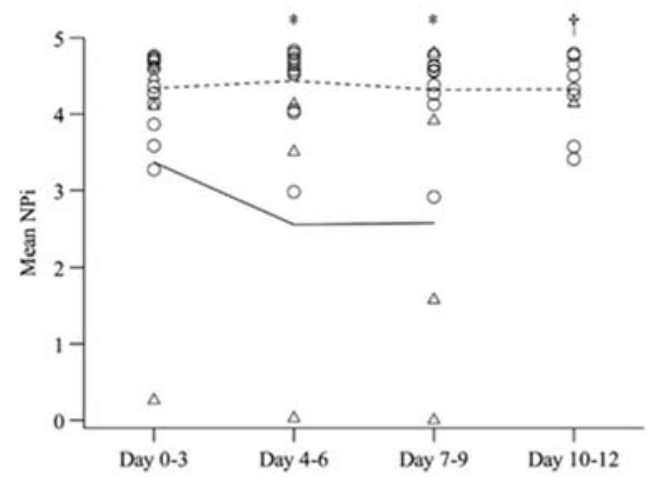

E

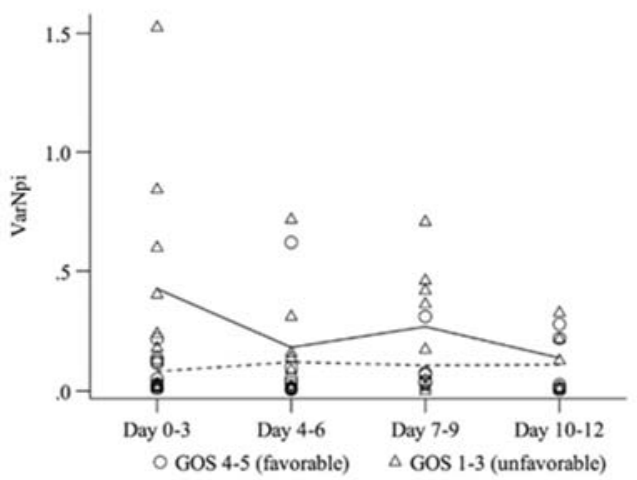

B

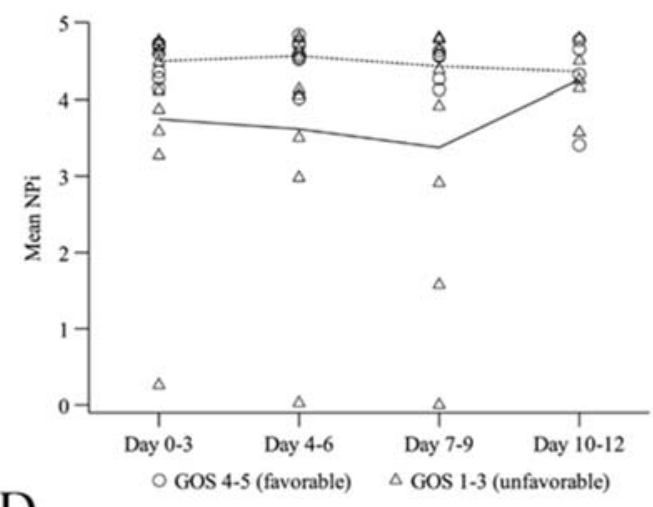

D

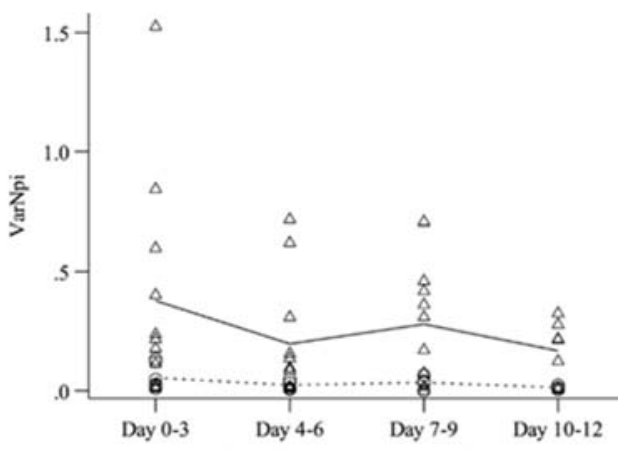

F

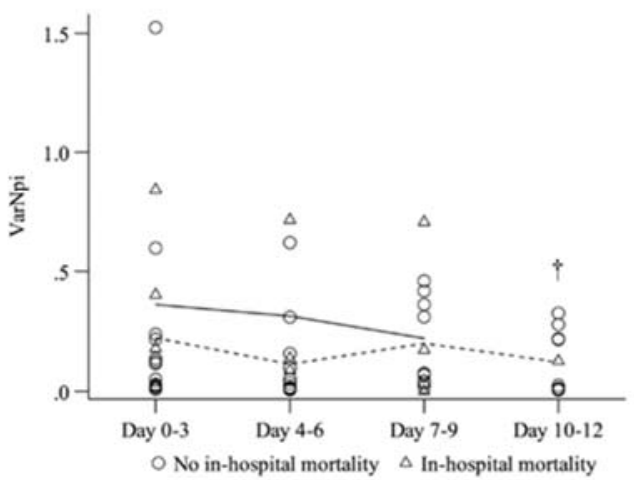


Figure 3. A) Spectral analysis of illustrative case 1 shows a high relative power in the circadian frequency band. B) Spectral analysis of illustrative case 2 shows a low relative power in the circadian frequency band, indicating a disruption of the circadian rhythm. C) The correlation between ICP ( $y$-axis) and NPi ( $x$-axis) shown as a scatter plot. A significant inverse correlation was found: Spearman's $r=-0.551, p<0.001$. D) Comparison of the relative power sum between patients with and without fatal outcome indicates a greater disturbance in the circadian rhythm in patients with fatal outcome.

A

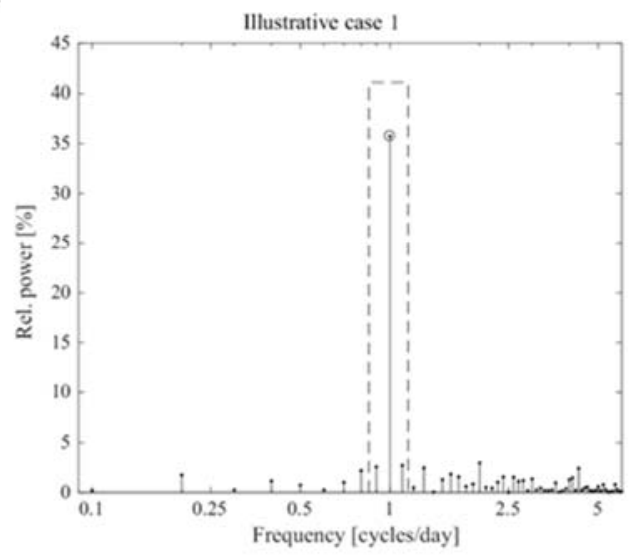

$\mathrm{C}$

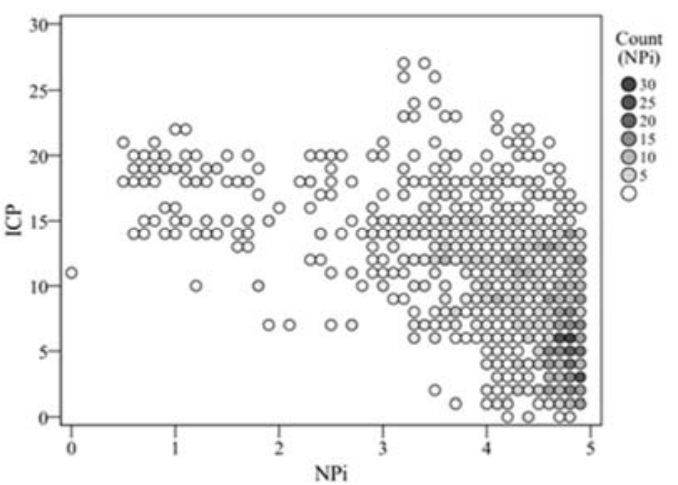

B

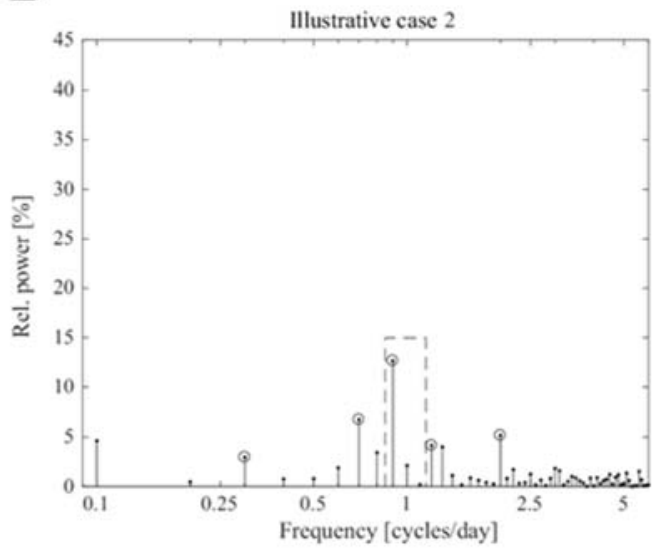

$\mathrm{D}$

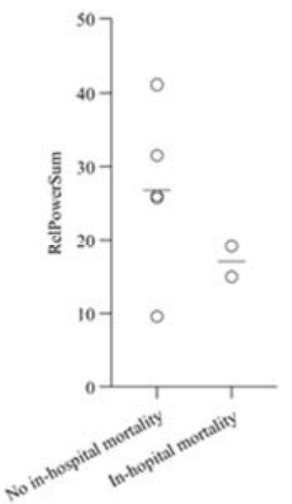

